\title{
Australian adolescent population norms for the child health utility index 9D—results from the young minds matter survey
}

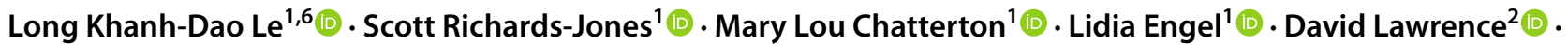

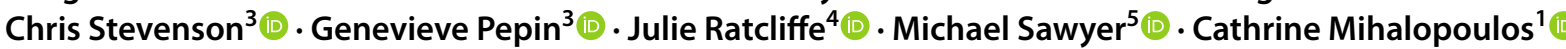

Accepted: 27 April 2021 / Published online: 17 May 2021

(C) The Author(s), under exclusive licence to Springer Nature Switzerland AG 2021

\begin{abstract}
Objective Patient-reported outcomes of health-related quality-of-life (HRQoL) are important descriptors of population health. A recent Australian adolescent population survey provided a unique opportunity to derive preference-based HRQoL. Methods Data from 2967 adolescents aged 11-17 years were analysed. An interviewer-led parent/carer questionnaire was administered for demographic variables and mental disorders of adolescents during previous 12 months using the Diagnostic Interview Schedule for Children. A self-report survey was administered to derive HRQoL using the child health utility nine-dimensions instrument (CHU-9D). Weighted HRQoL was derived for several demographic groups, mental disorder diagnosis, and youth risk behaviours.

Results The total population had a mean utility of 0.78 [standard deviation (SD): 0.20]. Males had a significantly higher mean utility $(0.81$, SD 0.18$)$ than females $(0.76$, SD: 0.21$)$ (Cohen's $d=0.23, p<0.001)$, and utility decreased with age for both males and females $(p<0.001)$. Family type and some parent/carer variables were associated with significant lower HRQoL scores with small effect size. Youth risk behaviours were associated with reduced HRQoL with moderate effect sizes. Adolescents who self-harmed, had suicidal ideation, or had a mental disorder had significantly lower utilities scores with moderate to large effect sizes compared to those who did not have such conditions.

Conclusions This study has provided contemporary Australian population norms for HRQoL in adolescents that may be used as cross comparison between studies as well as indicators allowing estimation of population health (e.g. estimation of the burden of disease) and can be used to populate future economic models.
\end{abstract}

Keywords Australia · Adolescents · CHU-9D · Quality-of-life · Utility scores · Quality-adjusted life year

\section{Plain English summary}

Long Khanh-Dao Le

long.le@deakin.edu.au

1 Deakin Health Economics, Faculty of Health, Institute for Health Transformation, School of Health and Social Development, Deakin University, Geelong, Australia

2 Graduate School of Education, The University of Western Australia, Perth, Australia

3 Faculty of Health, School of Health and Social Development, Deakin University, Geelong, Australia

4 Health and Social Care Economics Group, Caring Futures Institute, Flinders University, Adelaide, Australia

5 School of Psychology and Adelaide Medical School, University of Adelaide, Adelaide, SA, Australia

6 School of Health and Social Development, Deakin University, Geelong, Victoria, Australia
Patient-reported outcomes of health-related quality-of-life (HRQoL) are important descriptors of population health. A lack of recent estimates of the HRQoL for the Australian adolescent population for both demographic and mental disorder diagnostic groups means that there is an important gap in the literature. A recent Australian adolescent population survey provided a unique opportunity to derive preferencebased HRQoL for these groups in the Australian adolescent population. Data from 2967 adolescents aged 11-17 years were analysed. Family type and some parent/carer variables were associated with lower HRQoL in adolescents. Adolescents who used tobacco, alcohol, cannabis and other drug, self-harmed, had suicidal ideation, or had a mental disorder had significantly lower HRQoL compared to those who did not use these agents or have such conditions. This study 
has provided contemporary Australian population norms for HRQoL in adolescents that may be used as cross comparison between studies as well as indicators allowing estimation of population health (e.g. estimation of the burden of disease) and can be used to populate future economic models to evaluate interventions for mental health and wellbeing.

\section{Introduction}

Population norms for patient-reported outcome (PRO) measures are useful tools in helping to understand and improve population health. These norms represent the average values for a given PRO health instrument by age and gender and have many potential uses [1]. They also help to provide a standardised measure of population health to contextualise results from other studies, and a reference against which clinical treatment and public healthcare program outputs can be evaluated [2]. Population norms for demographic sub-populations (such as socio-economic groups or ethnic groups) can also be derived if appropriate data sources to categorise these groups are available. As such, population norms can be useful in comparing the health of heterogeneous demographic groups [3] and in identifying health inequalities [2]. Examining PRO measures for mental disorder diagnostic groups helps quantify the burden of disease associated with specific disorders [4].

PRO measures can also play an important role in resource allocation decisions by providing advice to policy-makers on the value-for-money credentials of healthcare interventions. Recognising the importance of improving population life expectancy while also ensuring good levels of health-related quality-of-life (HRQoL) has led to the development of the quality-adjusted life year (QALY), which combines mortality and morbidity into a single metric [5]. When deriving QALYs, HRQoL is often measured using preference-based measures, also known as multi-attribute utility instruments (MAUIs) [5]. MAUIs are essentially HRQoL questionnaires with an added scoring algorithm that provides a utility value for different health states as described by the questionnaire. Utility values are widely used and are measured on a scale of $0-1$ where 0 is equivalent to being dead and 1 is considered perfect health [5]. Utility values constitute the HRQoL component (i.e. morbidity component) of QALYs. QALYs are also commonly used as the health outcome measure in cost-utility analysis (CUA) that inform public healthcare funding decisions internationally [6]. In this way, PRO measures are intrinsically incorporated within value-for-money evidence used to help inform healthcare resource allocation funding decisions both within Australia and internationally.
Contemporary, HRQoL population norms are important given that the continually changing sociodemographic profile of Australia means historical norms can quickly become out-dated [2]. Likewise, up-to-date HRQoL values for mental disorder diagnostic groups are also important within the context of changing population characteristics and external influences, but also changes in available health care over time. HRQoL can be measured in young people through the use of MAUIs developed and validated in child and adolescent populations, such as the child health utility nine-dimensions (CHU-9D) [7]. Whilst recent high-quality studies exist for the HRQoL population norms of Australian adults [using the assessment of quality-of-life (AQoL-4D) and the short form six dimension (SF-6D) MAUIs] [1, 2, 8], population norms data for HRQoL in the Australian child and adolescent population are sparse. Petersen et al. provided an empirical comparison of the CHU-9D and the pediatric quality-of-life inventory (PedsQL) 4.0 Short Form in an Australian adolescent (15-17-year old) sample $(n=775)$ and subsequently provided a mean utility estimate for the sample. Chen et al. compared the performance of the CHU-9D with the childfriendly EQ-5D version (EQ-5D-Y) MAUI in an Australian adolescent sample $(n=2020)$ and provided CHU-9D and EQ$5 \mathrm{D}-\mathrm{Y}$ utility estimates for the population, as well as by sex and for two separate age groups (under 15 years and over and equal 15 years) [9]. Catchpool et al. provided recent utility estimates by sex but only for Australian children aged 11-12 years old [10]. These results are useful; however, estimates from sub-populations, for example, different socio-economic demographic groups, are lacking, and estimates that are representative across the entire Australian adolescent population are needed.

The Second Australian Child and Adolescent Survey of Mental Health and Wellbeing survey [the Young Minds Matter (YMM) survey] included robust sampling methodology, a large sample size, extensive information about the demographic characteristics of participating adolescents and their families, and a structured parent interview used to identify mental disorders being experienced by participating adolescents[11]. The survey also included the CHU-9D for the youth cohort who are 11-17-year-old adolescents [11]. The data from the YMM survey provide an excellent opportunity to derive HRQoL estimates for population norms and mental disorder diagnostic groups that are representative of the entire Australian adolescent population.

The objective of this study was to derive population norms for Australian adolescents aged 11-17 years by demographic characteristics, behavioural risk factors, and mental health diagnosis utilising CHU-9D utility data from the recent Young Minds Matter survey. 


\section{Methods}

\section{Young minds matter survey methods and participants}

Characteristics and methodology of the survey have been described in detail in a previous publication [11]. Briefly, the young minds matter survey was a national household survey conducted from 2013-2014 with the aim of capturing nationally representative data on the mental health and wellbeing of Australian children and adolescents [11]. A sample of 5500 4-17-year-olds was randomly selected from the Australian population through a multi-stage area-based sampling procedure. A sample of small geographic areas [in this survey the Statistical Area 1 or SA1 area, designed by the Australian Bureau of Statistics (ABS)] was selected foe both the main sample and oversample. A total of 266 SA1 areas (excluding SA1 areas in very remote areas) were selected from those where there were at least ten families with children living at the time of the 2011 Census of Population and Housing conducted by the ABS. Interviewers went door to door in randomly selected areas to identify families with children aged 4-17 years [11]. In households where there was more than one child, a child was selected at random by a computer. An oversample of 800 16-17-year-olds were additionally included as this is a high risk period for onset of mental disorders and risk behaviours, and increase in independent help seeking in this age group [12]. Previous analyses of this survey indicated the sample to be representative of Australian children and adolescents aged 4-17 years in terms of the age and gender of the child, family structure, parental education, income and employment, housing tenure and country of birth of both children and adolescents and their parents and carers [13].

Face-to-face interviews were conducted with parents/ carers including demographic questions and DISC-IV modules. The DISC-IV is a standardised diagnostic instrument developed under the auspices of the United States National Institute of Mental Health, which is used worldwide to assess mental disorders in large samples. Parents and carers were asked for their consent to approach the adolescent to as whether he or she would complete a youth self-report questionnaire [14]. Paper consent forms were completed by both parents or carers and young people at the completion of the household interviews [14]. Where the selected child was aged 11 years or older, adolescents were invited to complete a self-report questionnaire on a table computer including questions about risk behaviours, bullying and the CHU-9D instrument [12]. The overall response rate for households in the survey was 55\% (6310 eligible households participated) [11]. In participating households, $89 \%$ of eligible adolescents $(n=2967)$ completed the self-report survey [11].
The survey received ethical approval from the Australian Government Department of Health Human Research Ethics Committee and the University of Western Australia Human Research Ethic Committee.

\section{Measures}

Demographic and clinical measures for adolescents included: age (by year); sex; family type (or family blending based on relationships between household members); geographical remoteness (a measure of a physical location's level of access to goods and services, e.g. the smallest area SA1 for which census data are available, with a population between 200 and 800 and average size 400 people [15]); marital status of parent/carer(s); a general socio-economic index [index of relative socio-economic disadvantage (IRSD) that summarises a range of information about the economic and social conditions of people [15]]; equivalised pre-tax household income [whereby household income is adjusted for the size and composition of the household based on the modified organisation for economic co-operation and development (OECD) equivalence scale]; whether the adolescent was bullied in the previous year; carer work force participation; carer(s) drinking status; carer(s) smoking status; carer(s) education status; and, carer(s) previous mental diagnosis status. All of these variables were included in the parent interview dataset except for bullying status, which was included in the adolescent self-report survey. For variables with multiple categories of answers (e.g. carer(s) drinking frequency), dichotomous categories were manually created during analyses.

Adolescents completed the CHU-9D, which is a validated self-report preference-based measure of HRQoL suitable for self-completion in individuals aged 7-17 years $[7,16,17]$. It includes nine health dimensions (worry, sadness, pain, tiredness, annoyance, school work/homework, sleep, daily routine, ability to join in activities) with five levels per dimension [7]. A published utility scoring algorithm was used which was based on best-worst scaling scores for CHU-9D health states elicited from Australian adolescents (with the best-worst scaling scores re-scaled onto the 0-1 QALY scale by empirical mapping to time trade-off values for a series of CHU-9D health states ranging from mild to severe impairment states from young Australian adults aged 18-29 years) [18]. Possible utility scores from the CHU-9D scoring algorithm range from 1.00 (perfect health) to zero (being dead), although negative values are also possible which denote health states measured by the CHU-9D instrument that are considered worse than being dead (e.g. - 0.1059) [18]). 


\section{Behavioural risk factors}

Adolescents were asked if they had ever used tobacco, alcohol, cannabis, or other drugs. To assess lifetime self-harm, a question regarding deliberate harm or injury, with or without suicidal intent was asked. An additional question assessed suicidal ideation in the past 12 months. Bullying status was assessed by a single self-reported question whether adolescents have been involved in victimisation and/or perpetration of bullying.

\section{Mental disorders}

Mental disorders were assessed using specific diagnostic modules from the Diagnostic Interview Schedule for Children Version IV (DISC-IV) and a specifically developed Impact on Functioning module [19]. Disorder status is determined according to the criteria of the Diagnostic and Statistical Manual of Mental Disorders Version IV (DSM-IV), which is the diagnostic classification system used clinically worldwide. DISC-IV modules for four main disorders were included in the survey: Anxiety disorders including social phobia, separation anxiety disorder, generalised anxiety disorder and obsessive-compulsive disorder; Major depressive disorder; attention-deficit/hyperactivity disorder (ADHD); and conduct disorder.

\section{Statistical analyses}

To ensure the derived utilities from this study were representative of the Australian 11-17-year-old resident population based on 30th June 2013 Australian Bureau of Statistics (ABS) estimates, population weights based on age, sex, household income and family size provided in the survey data were applied to utility estimates [10]. The weighted mean, median, standard deviation (SD) and $95 \%$ confidence intervals (CIs) of the utility values were reported. Tests for statistically significant differences in utility values at the 5\% significant level were conducted using the Adjusted Wald test with Bonferroni adjustment (due to multiple comparisons). Effect sizes were calculated using Cohen's $d$, whereby an effect size of $0.2,0.5$ and $\geq 0.8$ are interpreted as representing a small, moderate or large effects ,respectively [2, 20]. The proportions of total individuals, and the proportion of individuals by sex, within each utility decile were graphically plotted.

Missing data (i.e. 'not stated' or 'don't know') were present in $<0.2 \%$ of the sample for bullying status, carer drinking and carer smoking variables. Data rules were employed for these three variables assuming these adolescents with missing data belonged to the higher risk category for the variable (e.g. was bullied in the previous year). Overall, $4.85 \%$ of the sample had missing data (i.e. 'not stated') for the equivalised household income variable, therefore utility estimates for these individuals were not calculated for this variable, rather utility values for this variable were calculated on a complete case basis. No other missing data were present from the survey responses.

Stata SE Version 15 was used to conduct analyses as well as to graphically plot utility decile proportions.

\section{Results}

The mean sample age was 14.6 (SD 2.0) years. Nearly half of the sample (48.5\%) were female. The CHU-9D population norms for the total 11-17-year-old population, and by gender and age, are shown in Table 1 . The mean utility for the total population was 0.78 (95\% CI 0.78-0.79), with the mean utility of males [0.81 (95\% CI $0.80-0.82)]$ being statistically significantly $(p<0.001)$ higher than the mean utility of females [0.76 (95\% CI 0.75-0.77)]. Eleven-yearold females had significantly higher utility values than males ( 0.86 vs. $0.82 p<0.05$ ). This trend reversed after age 14 , when males had significantly higher utility values $(0.73 \mathrm{vs}$. $0.80 p<0.05)$. That significant trend of higher utility values for males then continued to age 17 .

Figure 1 displays CHU-9D scores by age and sex. A similar trend for both males and females was observed, with decreasing utility scores by age. The 5 th centile is notably lower for females, indicating lower CHU-9D scores across all age groups.

Population norms by other demographic variables are shown in Table 2. For the total population, utility values were significantly higher for two-parent families $(d=0.20$, $p<0.001)$, families with both carers working $(p<0.05)$ and where a carer had never been diagnosed with a mental disorder $(d=0.17, p<0.001)$. These effects were consistent across sex with the exception that no significant difference of utility scores was found among work force participation in female carers. No statistical differences were observed for the level of remoteness, IRSD quintiles, household income and carer education level.

The CHU-9D utility values for youth risk behaviours and mental diagnosis during lifetime or 12 months are shown in Table 3. Adolescents, regardless of gender who have ever used tobacco, alcohol, cannabis, or other drugs had significantly lower utility scores with moderate effect sizes (effect size ranging from 0.40 to 0.66 ) compared to those who have not used such substances. For self-harm (during lifetime) and suicidal ideation (during the last 12 months), the utility losses were represented by large effect sizes $(d=1.38-1.39)$ for those aged over 12 years with syndromes. Adolescents who were victims or perpetrators of bullying during the last 12 months showed similar impairment on the CHU9D utility scores with moderate effect sizes (i.e. bullying 
Table 1 Weighted CHU-9D utility scores for the total population, and by age and sex

\begin{tabular}{|c|c|c|c|c|c|c|c|}
\hline \multirow[t]{2}{*}{ Age (year) } & \multirow[t]{2}{*}{ Sex } & \multirow[t]{2}{*}{$n(\%)^{\mathrm{a}}$} & \multicolumn{5}{|c|}{ Population-weighted CHU-9D utility scores } \\
\hline & & & Mean & SD & Median & $95 \% \mathrm{CI}$ & Statistics $\mathrm{b} /$ effect size \\
\hline \multirow[t]{3}{*}{11} & All & $312(14.0)$ & 0.84 & 0.16 & 0.88 & $0.82-0.86$ & Between sex \\
\hline & Male & $161(52.0)$ & 0.82 & 0.17 & 0.86 & $0.80-0.85$ & $p<0.05$ \\
\hline & Female & $151(48.0)$ & 0.86 & 0.15 & 0.89 & $0.84-0.88$ & Cohen's $d=1.09$ \\
\hline \multirow[t]{3}{*}{12} & All & $341(14.1)$ & 0.82 & 0.17 & 0.88 & $0.80-0.84$ & Between sex \\
\hline & Male & $177(52.2)$ & 0.82 & 0.18 & 0.88 & $0.80-0.85$ & $p=0.77$ \\
\hline & Female & $164(47.8)$ & 0.82 & 0.17 & 0.87 & $0.79-0.84$ & Cohen's $d=0.03$ \\
\hline \multirow[t]{3}{*}{13} & All & $310(14.2)$ & 0.8 & 0.18 & 0.86 & $0.78-0.82$ & Between sex \\
\hline & Male & $163(50.7)$ & 0.81 & 0.17 & 0.87 & $0.78-0.84$ & $p=0.31$ \\
\hline & Female & $147(49.3)$ & 0.79 & 0.2 & 0.85 & $0.76-0.82$ & Cohen's $d=0.11$ \\
\hline \multirow[t]{3}{*}{14} & All & $343(14.4)$ & 0.77 & 0.21 & 0.82 & $0.75-0.79$ & Between sex \\
\hline & Male & $183(53.7)$ & 0.8 & 0.18 & 0.86 & $0.77-0.82$ & $p<0.05$ \\
\hline & Female & $160(46.3)$ & 0.73 & 0.24 & 0.78 & $0.70-0.77$ & Cohen's $d=0.30$ \\
\hline \multirow[t]{3}{*}{15} & All & $309(14.1)$ & 0.76 & 0.2 & 0.8 & $0.73-0.78$ & Between sex \\
\hline & Male & $168(54.9)$ & 0.8 & 0.18 & 0.85 & $0.77-0.83$ & $p<0.001$ \\
\hline & Female & $141(45.1)$ & 0.71 & 0.21 & 0.74 & $0.67-0.74$ & Cohen's $d=0.46$ \\
\hline \multirow[t]{3}{*}{16} & All & $718(14.5)$ & 0.74 & 0.22 & 0.79 & $0.73-0.76$ & Between sex \\
\hline & Male & $353(47.5)$ & 0.79 & 0.18 & 0.83 & $0.77-0.81$ & $p<0.001$ \\
\hline & Female & $365(52.5)$ & 0.69 & 0.23 & 0.74 & $0.67-0.72$ & Cohen's $d=0.48$ \\
\hline \multirow[t]{3}{*}{17} & All & $634(14.6)$ & 0.76 & 0.21 & 0.81 & $0.74-0.78$ & Between sex \\
\hline & Male & 325 (49.7) & 0.79 & 0.18 & 0.83 & $0.77-0.81$ & $p<0.001$ \\
\hline & Female & $309(50.3)$ & 0.73 & 0.23 & 0.79 & $0.70-0.75$ & Cohen's $d=0.33$ \\
\hline \multirow[t]{3}{*}{ Total } & All & 2967 (100) & 0.78 & 0.2 & 0.84 & $0.78-0.79$ & Between sex \\
\hline & Male & $1530(51.5)$ & 0.81 & 0.18 & 0.86 & $0.80-0.82$ & $p<0.001$ \\
\hline & Female & $1437(48.5)$ & 0.76 & 0.21 & 0.82 & $0.75-0.77$ & Cohen's $d=0.23$ \\
\hline
\end{tabular}

$n$ number in sample, $C H U-9 D$ child health utility instrument nine-dimensions, $S D$ standard deviation, $C I$ confidence interval

${ }^{\mathrm{a}} n$ refers to sample size, $\%$ refers to proportion in population with population weights applied

${ }^{\mathrm{b}}$ Adjusted-Wald test using Bonferroni adjustment perpetration $d=0.43, p<0.001$ and bullying victimisation $d=0.59, p<0.001)$. Adolescents with mental disorder diagnosis presented a utility loss with moderate effect size $(d=0.53, p<0.001)$ compared to those who did not have a mental disorder. Specifically, lower utility scores were found for anxiety disorders $(d=0.45, p<0.001)$, major depressive disorder $(d=0.76, p<0.001)$ and ADHD $(d=0.23$, $p<0.05)$. Due to small sample size for conduct disorder, there was no significant difference of utility scores in the male and female subgroups but there was a statistically significant difference for the overall group of $\mathrm{CD}(d=0.37$, $p<0.05)$.

\section{Discussion}

This study provides utility estimates to describe the health of the Australian adolescent population. These values provide a benchmark for comparison to other adolescent populations and are important for use in economic models evaluating interventions for this age group. The mean utility for the population (0.78) indicates that HRQoL for the Australian adolescents aged 11-17 years was relatively high, which is to be expected given the age range. The mean utility score in this study was somewhat similar compared to scores reported in a previous community survey in the same population (0.78 vs. 0.80) [9] and higher compared to scores reported for younger clinical samples (mean age 11.7 years) (0.78 vs. 0.73) [21]. It is noted that to derive CHU-9D utility scores, this study used an Australian adolescent-specific scoring algorithm, while Chen et al. [9] used an Australian adult general population-specific scoring algorithm. Previous research indicated that adults, in general, placed less weight upon impairments in mental health (worried, sad, annoyed) and more weight upon moderate to severe levels of pain relative to adolescents [22]. The source of valuation is an important consideration, where a previous study found that adults place less weight upon impairment 


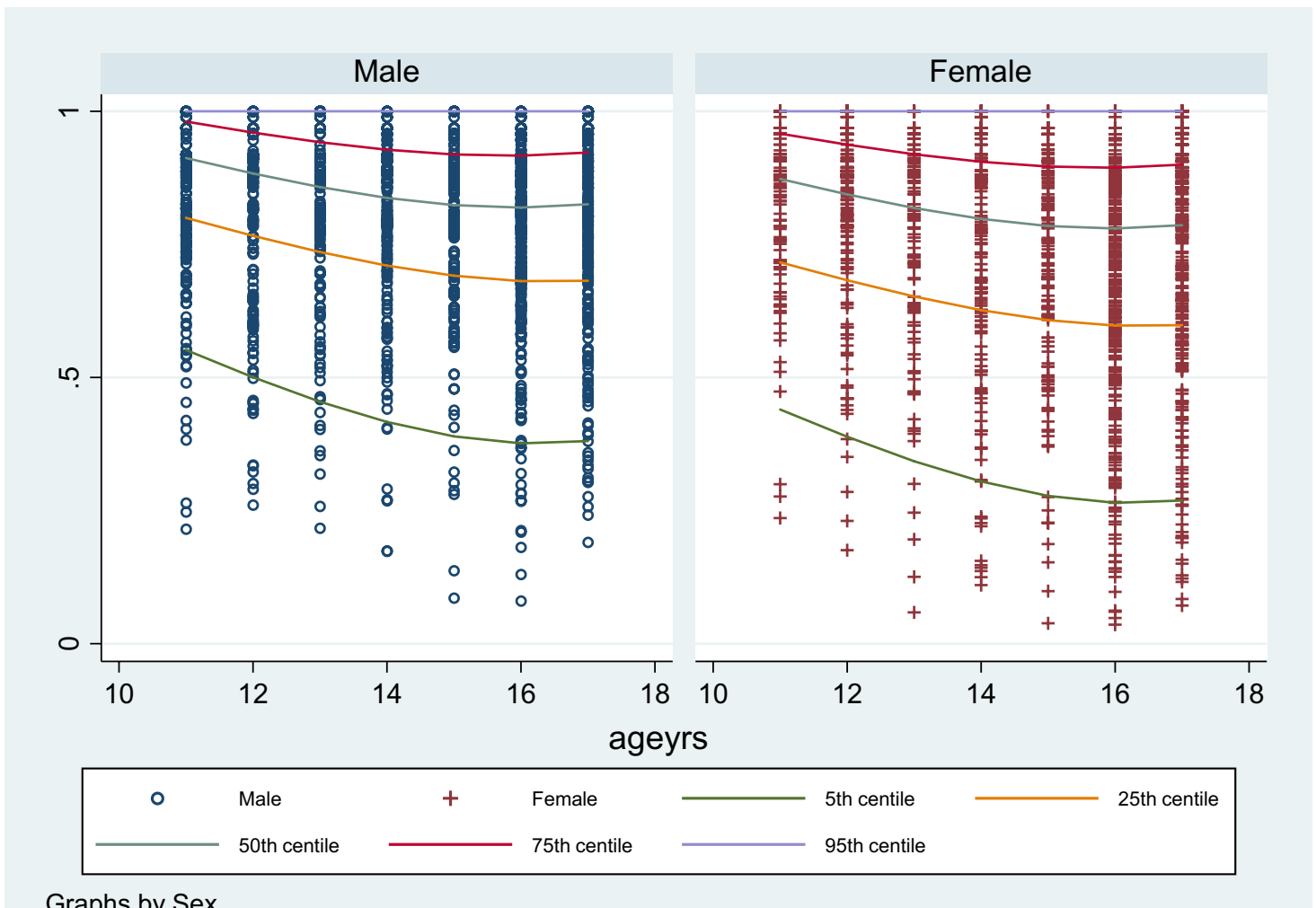

Graphs by Sex

Fig. 1 Proportions of individuals in CHU-9D utility centiles by sex and age. $C H U-9 D$ child health utility instrument nine-dimensions

in mental health and more weight upon moderate to severe levels of pain, relative to adolescents [17]. The mean utility for adolescents aged 12 years $(0.82 ; 95 \%$ CI $0.80-0.84)$ was comparable to the mean utility of Australian adolescents aged 12 years $(0.81$; CI $0.81-0.82)$, recently reported from CHU-9D estimates in Catchpool et al. [10], which used a similar scoring algorithm as applied in this study.

The results showed that adolescent males aged 11-17 years had significantly higher mean utility scores than females, and this was largely due to the significantly higher mean utilities in males aged from 14 to 17 years. This finding is consistent with previous literature that found lower utility scores in females compared to males, except for those aged 11 years $[9,10]$. The results also indicate that adolescents under 15 years had lower utility scores compared to those above or equal to 15 years, which is in line with findings reported by Chen et al. [9].

A number of demographic factors appeared to be significantly associated with adolescents' HRQoL. However, these results need to be interpreted in caution given that these effect sizes were small. Results of this study indicate that the mental health of parent/carers may impact on the HRQoL of their children. Previous research showed that interventions that included cognitive, behavioural, or psychoeducational components to reduce mental disorders in parents were able to decrease risk of developing mental disorders by $40 \%$ in the offspring of parents [23]. Parents/Carers employment was also positively associated with adolescent HRQoL, although this was only true for the male adolescent population. This suggests that sex may be a confounding factor in this relationship, with males' HRQoL being more heavily impacted by the work force participation of their carers. Although geographical remoteness was not shown to have a significant relationship with HRQoL, the mean utility score for adolescents in remote areas of Australia was numerically lower than adolescents from other geographical areas. Carer drinking frequency had a significant relationship with HRQoL for males but not females, suggesting that gender may be a confounding factor in this relationship, with males much more heavily impacted by carer alcohol consumption than females.

Consistent with other published literature, adolescents who reported being bullied or perpetrators of bullying had a substantially lower HRQoL, possibly indicating that the psychological and physical impacts of bullying are noticeably diminishing Australian adolescents' HRQoL [24-26]. Compared to male adolescents, more female adolescents reported being bullied, while fewer female adolescents 


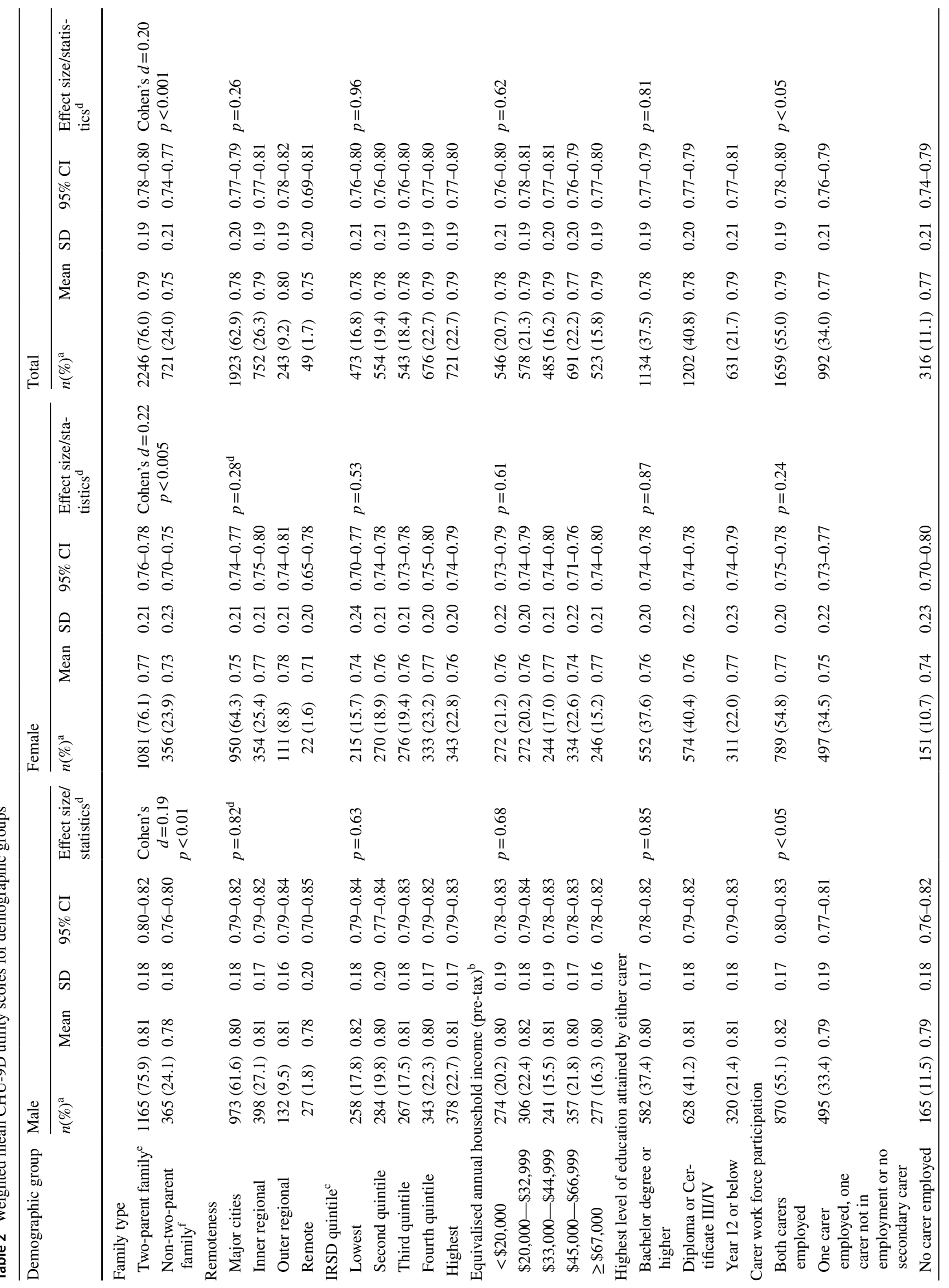




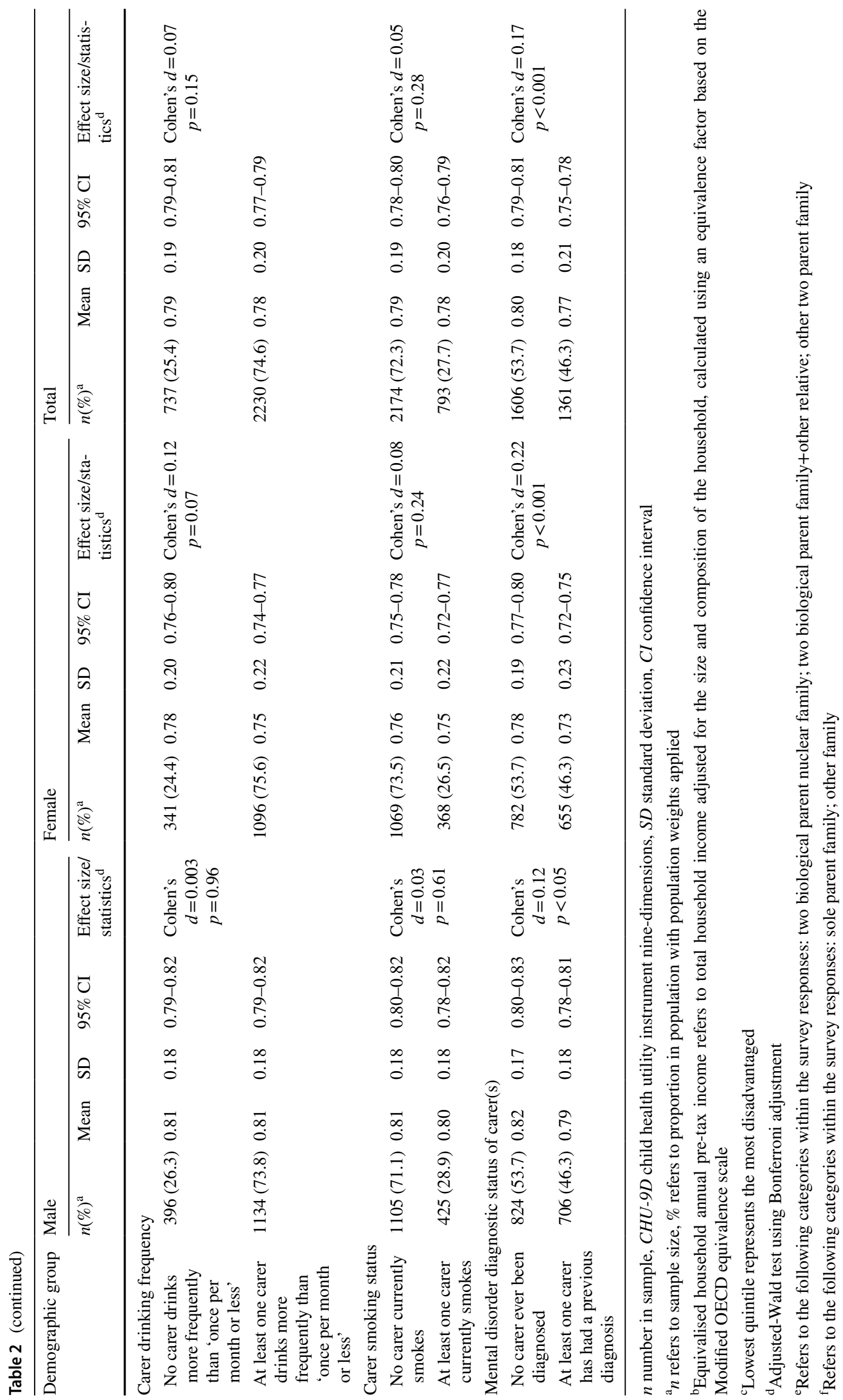




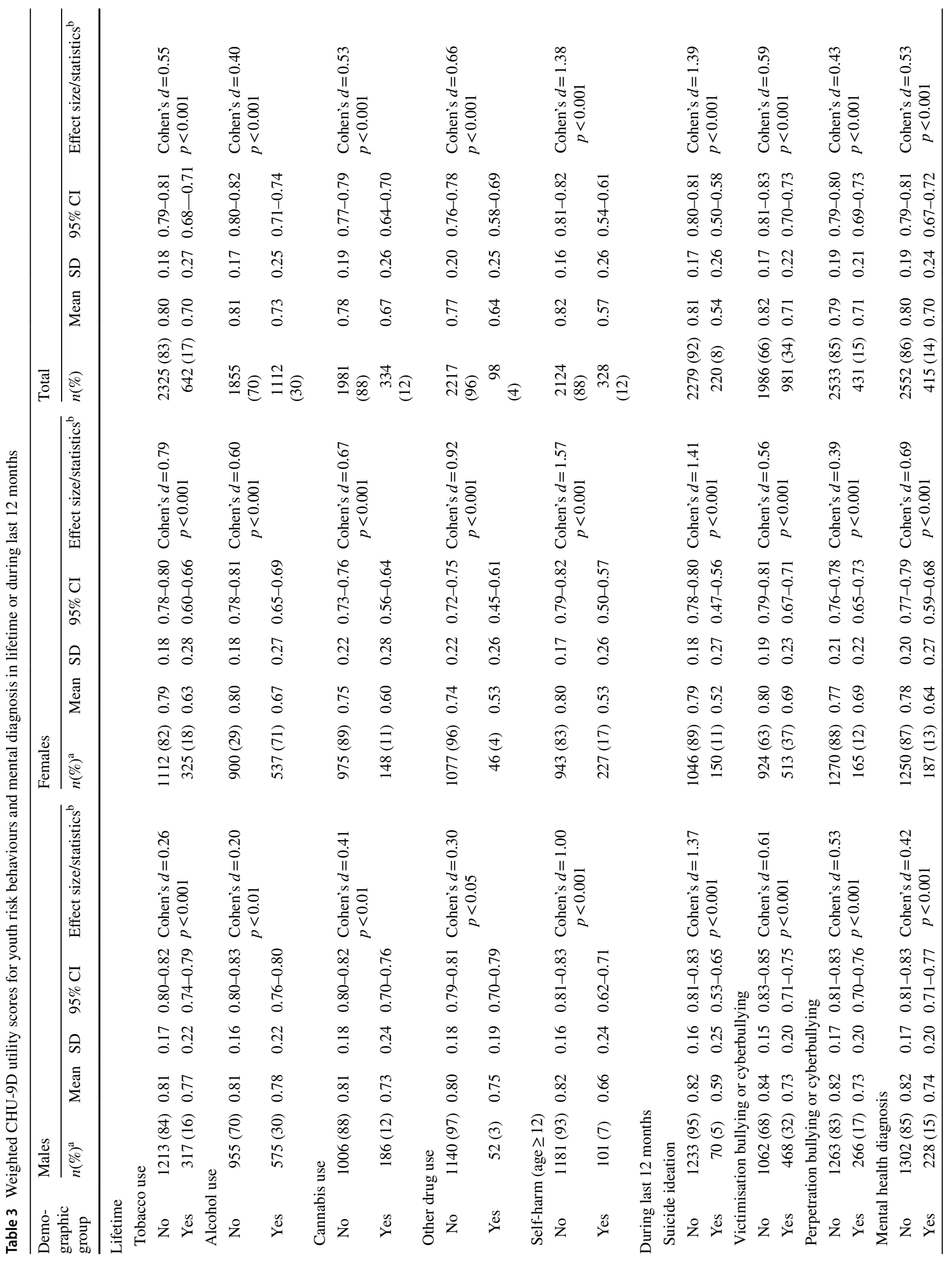


reported being perpetrators of bullying. The HRQoL for females being bullied or being perpetrators was also lower than in males (although both bullying status was highly significant in both males and females). It is unclear whether this is due to a higher prevalence and impact of bullying in female adolescents, or due to females being more willing to report being bullied and its impacts.

With respect to mental disorder diagnoses, adolescents with a diagnosis of a mental disorder, including major depressive disorder, ADHD, and anxiety disorders, had significantly lower HRQoL compared to those without a mental disorder. Given that the COVID-19 pandemic has worsened the already worrying mental health statistics, it is vital for further research to explore the impact of severity of mental disorders on HRQoL in adolescents. This is the first study to report utility scores for adolescents with lifetime selfharm or 12-month suicide ideation who were found greatest impact on HRQoL. The utility values for self-harm and suicide are particularly useful for economic modelling the effect of interventions to prevent these issues.

\section{Strength and limitations}

This study has several strengths, such as delineating the findings based on a nationally representative sample of the Australian adolescent general population. The majority of demographic and clinical characteristics in this study were self-reported by adolescents, enabling interpretations of data from the adolescents' point of view rather than proxy report. However, it is noted that mental disorder diagnosis of adolescents were reported by their parents/carers, which might conflict with self-report data. Given that the utility scores were only collected in those aged 11-17, this study is not able to explore the HRQoL in younger children (under 11 years). Recall and the length of time covered by the questionnaires may also impact results. For example the short recall period of today of CHU-9D may limit its applicability as it may not be sufficiently sensitive to capture issues that irregularly affect respondents [24]. Furthermore, different MAUIs include different domains of HRQoL and have adopted different techniques in developing the scoring algorithms. Therefore, the results of the current study may not be directly comparable to surveys that have used a different MAUI. There is a need for future surveys to understand how sociodemographic profile changes affect the population norms of utility scores.

\section{Conclusions}

Australian adolescents have an overall high level of HRQoL, which slightly decreases through adolescence and is higher in males. Family structure, carer mental disorder diagnostic 
status and carer working status are demographic variables, which appear to be related to adolescents' HRQoL. Risky behaviour and mental diagnoses are also related to significantly reduced HRQoL of adolescents. Results from this study can be used for benchmarking comparisons of other population groups/patient samples as well as for economic modelling of interventions to improve mental health and wellbeing potentially.

Acknowledgements We would like to acknowledge the Deakin School of Health and Social Development for funding this research and the Telethon Kids Institute and University of Western Australia for originally conducting the population survey on which this study is based. LK-DL has been funded by the Alfred Deakin Postdoc Research Fellow and NHMRC Centre of Research Excellence in Prevention and Early Intervention in Mental Illness and Substance Use (PREMISE).

Data availability The de-identified data are available to any interested researchers through the Australian Data Archive who need to be approved by their Institution's ethics committee or Institutional Review Board. Full information on how to apply for access to the data set is available on our website www.youngmindsmatter.org.au under the resources tab.

\section{Declarations}

Conflict of interest The authors declared no conflict of interest

Ethical approval The survey received ethical approval from the Australian Government Department of Health Human Research Ethics Committee and the University of Western Australia Human Research Ethic Committee. Parents and carers were asked for their consent to approach the adolescent to as whether he or she would complete a youth self-report questionnaire. Paper consent forms were completed by both parents or carers and young people at the completion of the household interviews.

\section{References}

1. Maxwell, A., Özmen, M., Iezzi, A., \& Richardson, J. (2016). Deriving population norms for the AQoL-6D and AQoL-8D multi-attribute utility instruments from web-based data. Quality of Life Research, 25(12), 3209-3219

2. Hawthorne, G., Korn, S., \& Richardson, J. (2013). Population norms for the AQoL derived from the 2007 Australian national survey of mental health and wellbeing. Australian and New Zealand Journal of Public Health, 37(1), 7-16

3. Richardson, J., Khan, M.A, Chen, G., Iezzi A., Maxwell, A. (2012). Population norms and Australian profile using the Assessment of Quality of Life (AQoL) 8D utility instrument. Centre for Health Economics Research Paper

4. Mihalopoulos, C., Engel, L., Le, L.K.-D., Magnus, A., Harris, M., \& Chatterton, M. L. (2018). Health state utility values of high prevalence mental disorders in Australia: results from the national survey of mental health and wellbeing. Quality of Life Research, 27(7), 1815-1825

5. Drummond, M. F., Sculpher, M. J., Torrance, G. W., O'Brien, B., \& Stoddart, G. (2015). Methods for the economic evaluation of health care programmes. (4th ed.). Oxford University Press.
6. Whitty, J. A., \& Littlejohns, P. (2015). Social values and health priority setting in Australia: an analysis applied to the context of health technology assessment. Health Policy, 119(2), 127-136

7. Ratcliffe, J., Stevens, K., Flynn, T., Brazier, J., \& Sawyer, M. (2012). An assessment of the construct validity of the CHU9D in the Australian adolescent general population. Quality of Life Research, 21(4), 717-725

8. Norman, R., Church, J., van den Berg, B., \& Goodall, S. (2013). Australian health-related quality of life population norms derived from the SF-6D. Australian and New Zealand Journal of Public Health, 37(1), 17-23

9. Chen, G., Flynn, T., Stevens, K., Brazier, J., Huynh, E., Sawyer, M., et al. (2015). Assessing the health-related quality of life of Australian adolescents: an empirical comparison of the child health utility 9D and EQ-5D-Y instruments. Value Health., 18(4), 432-438

10. Catchpool, M., Gold, L., Grobler, A. C., Clifford, S. A., \& Wake, M. (2019). Health-related quality of life: population epidemiology and concordance in Australian children aged 11-12 years and their parents. British Medical Journal Open, 9(Suppl 3), 157-164

11. Hafekost, J., Lawrence, D., de BoterhovenHaan, K., Johnson, S. E., Saw, S., Buckingham, W. J., et al. (2016). Methodology of young minds matter: The second Australian child and adolescent survey of mental health and wellbeing. Australian \& New Zealand Journal of Psychiatry., 50(9), 866-875

12. Hafekost, J., Johnson, S., Lawrence, D., Sawyer, M., Ainley, J., Mihalopoulos, C., et al. (2016). Introducing 'young minds matter.' The Australian Economic Review, 49(4), 503-514

13. Lawrence, D., Johnson, S., Hafekost, J., de BoterhovenHaan, K., Sawyer, M., Ainley, J., et al. (2015). The mental health of children and adolescents: Report on the second Australian child and adolescent survey of mental health and wellbeing. Department of Health.

14. Telethon Kids Institute. (2015). Young minds matter: The second Australian child and adolescent survey of mental health and wellbeing, survey user's guide. Centre for Child Health Research.

15. Statistics ABO. (2011). Census of population and housing: Socio-economic indexes for areas (SEIFA). Australian Bureau of Statistics.

16. Stevens, K., \& Ratcliffe, J. (2012). Measuring and valuing health benefits for economic evaluation in adolescence: An assessment of the practicality and validity of the child health utility 9D in the Australian adolescent population. Value Health, 15(8), 1092-1099

17. Ratcliffe, J., Stevens, K., Flynn, T., Brazier, J., \& Sawyer, M. G. (2012). Whose values in health? An empirical comparison of the application of adolescent and adult values for the CHU-9D and AQOL-6D in the Australian adolescent general population. Value Health, 15(5), 730-736

18. Ratcliffe, J., Huynh, E., Chen, G., Stevens, K., Swait, J., Brazier, J., et al. (2016). Valuing the child health utility 9D: Using profile case best worst scaling methods to develop a new adolescent specific scoring algorithm. Social Science and Medicine, 157, 48-59

19. Zubrick, S., Lawrence, D., Johnson, S., \& Hafekost, J. (2015). Measuring severity of mental disorders with the young minds matter parent/carer-reported impact items: Technical report. Centre for Child Health Research, The University of Western Australia.

20. Cohen, J. (1988). Statistical power analysis for the behavioural sciences. Hilldale.

21. Furber, G., \& Segal, L. (2015). The validity of the child health utility instrument (CHU9D) as a routine outcome measure for use in child and adolescent mental health services. Health and Quality of Life Outcomes, 13(1), 22

22. Ratcliffe, J., Huynh, E., Stevens, K., Brazier, J., Sawyer, M., \& Flynn, T. (2016). Nothing about us without us? A comparison of adolescent and adult health-state values for the child health 
utility-9D using profile case best-worst scaling. Health Economics, 25(4), 486-496

23. Siegenthaler, E., Munder, T., \& Egger, M. (2012). Effect of preventive interventions in mentally ill parents on the mental health of the offspring: Systematic review and meta-analysis. Journal of the American Academy of Child \& Adolescent Psychiatry, 51(1), 8-17

24. Fantaguzzi, C., Allen, E., Miners, A., Christie, D., Opondo, C., Sadique, Z., et al. (2018). Health-related quality of life associated with bullying and aggression: A cross-sectional study in English secondary schools. The European Journal of Health Economics, 19(5), 641-651
25. Beckman, L., Svensson, M., \& Frisén, A. (2016). Preferencebased health-related quality of life among victims of bullying. Quality of Life Research, 25(2), 303-309

26. Le, L.K.-D., Chatterton, M.L., Rapee, R., Fitzpatrick, S., Bussey, K., Hudson, J., et al. editors. (2019). Burden and preference-based quality of life associated with bullying. In 2019 World Congress on Health Economics. iHEA

Publisher's Note Springer Nature remains neutral with regard to jurisdictional claims in published maps and institutional affiliations. 